\title{
Fiber optic ultrasound transducers with carbon/PDMS composite coatings
}

Charles A. Mosse, Richard J. Colchester, Davinder S. Bhachu, Edward Z. Zhang, loannis Papakonstantinou, et al.

Charles A. Mosse, Richard J. Colchester, Davinder S. Bhachu, Edward Z. Zhang, loannis Papakonstantinou, Adrien E. Desjardins, "Fiber optic ultrasound transducers with carbon/PDMS composite coatings," Proc. SPIE 8943, Photons Plus Ultrasound: Imaging and Sensing 2014, 89430P (3 March 2014); doi: $10.1117 / 12.2037283$

SPIE. Event: SPIE BiOS, 2014, San Francisco, California, United States 


\title{
Fiber-optic Ultrasound Transducers with Carbon/PDMS Composite Coatings
}

\author{
Charles A. Mosse, ${ }^{a}$ Richard J. Colchester, ${ }^{a}$ Davinder S. Bhachu, ${ }^{b}$ Edward Z. Zhang, ${ }^{a}$ \\ Ioannis Papakonstantinou, ${ }^{\mathrm{c}}$ Adrien E. Desjardins ${ }^{\mathrm{a}}$ \\ aDepartment of Medical Physics and Bioengineering, University College London, \\ Malet Place Engineering Building, London, WC1E 6BT, United Kingdom \\ ${ }^{b}$ Materials Chemistry Centre, Department of Chemistry, University College London, \\ 20 Gordon Street, London, WC1H 0AJ, United Kingdom \\ 'Department of Electronic and Electrical Engineering, University College London, \\ Roberts Building, London, WC1E 7JE, United Kingdom
}

\begin{abstract}
Novel ultrasound transducers were created with a composite of carbon nanotubes (CNTs) and polydimethylsiloxane (PDMS) that was dip coated onto the end faces of optical fibers. The CNTs were functionalized with oleylamine to allow for their dissolution in xylene, a solvent of PDMS. Ultrasound pulses were generated by illuminating the composite coating with pulsed laser light. At distances of 2 to $16 \mathrm{~mm}$ from the end faces, ultrasound pressures ranged from 0.81 to $0.07 \mathrm{MPa}$ and from 0.27 to $0.03 \mathrm{MPa}$ with 105 and $200 \mu \mathrm{m}$ core fibers, respectively. Using an optical fiber hydrophone positioned adjacent to the coated $200 \mu \mathrm{m}$ core optical fiber, ultrasound reflectance measurements were obtained from the outer surface of a sheep heart ventricle. The results of this study suggest that ultrasound transducers that comprise optical fibers with CNT-PDMS composite coatings may be suitable for miniature medical imaging probes.
\end{abstract}

Keywords: Laser-generated ultrasound, photoacoustic, optical fiber, carbon nanotubes, PDMS

\section{INTRODUCTION}

Ultrasound imaging is widely used to guide minimally invasive procedures. For instance, intravascular ultrasound imaging is widely used to guide stent placements. Current-generation ultrasound imaging probes use piezoelectric transducers $^{1}$ or capacitive micro-machined ultrasonic transducers (CMUTs). ${ }^{2}$ Integration of these electrical transducers into medical devices can be challenging and expensive.

Recently there has been rapid progress with the development of optical transducers as alternatives to electrical transducers. Optical transducers generate ultrasound with the photoacoustic effect, in which absorption of pulsed or modulated light results in pressure waves. Several optical absorbers have been considered for generating ultrasound, including gold nanostructures, ${ }^{3}$ carbon black, ${ }^{4}$ graphite, ${ }^{5,6}$ graphene ${ }^{7}$ and carbon nanotubes (CNTs) ${ }^{8,9}$ The efficiency of the ultrasound generation process can be significantly increased with the inclusion of an elastomeric component such as polydimethylsiloxane (PDMS). ${ }^{3,4,8,9}$

With PDMS-CNT composites, micron-scale thicknesses are important for the generation of broadband ultrasound pulses. $^{8}$ Spin coating has been used to overlay a thin PDMS layer onto CNTs, thereby creating a PDMS-CNT composite. ${ }^{8}$ However, this method is not well suited to coating the distal ends of optical fibers, ${ }^{10}$ whereas dip coating is a method that is well suited for this purpose. Coating thicknesses of $10 \mu \mathrm{m}$ were reported by Kuroda and Nishino, in an application that involved coating the tapered ends of wires for use in scanning tunneling microscopy. ${ }^{11}$ To dip coat PDMS-CNT composites, a low viscosity mix of CNTs, PDMS, and a solvent is required, but dissolution of pristine CNTs is hindered by Van der Waals forces that result in aggregation. ${ }^{12}$

Photons Plus Ultrasound: Imaging and Sensing 2014, edited by Alexander A. Oraevsky, Lihong V. Wang, Proc. of SPIE Vol. 8943, 89430P · (C) 2014 SPIE · CCC code: 1605-7422/14/\$18 · doi: 10.1117/12.2037283 
The purpose of this study was to develop a novel method for creating a CNT-PDMS composite and to apply this composite to the end faces of optical fibers for ultrasound generation. One of the resulting optical transducers was used in conjunction with an optical fiber hydrophone to obtain ultrasound reflectance measurements of biological tissue $e x$ vivo.

\section{METHODS}

\subsection{Carbon nanotube functionalization}

Multiwall CNTs (724769, Sigma Aldrich, UK) with specified dimensions of 6-9 $\mathrm{nm} \times 5 \mu \mathrm{m}$ were functionalized with a method based on that described by Majeed et al. ${ }^{12}$ so that they could be dissolved in xylene, a solvent of PDMS. In this method, 1-pyrenebutyric acid $(3.5 \mathrm{mmol})$ and an excess amount of thionyl chloride were refluxed at $100{ }^{\circ} \mathrm{C}$ for 30 minutes in a round bottomed flask $(25 \mathrm{~mL})$. Unreacted $\mathrm{SOCl}_{2}$ was removed by distillation. The remaining solid pyrenebutyryl chloride was dissolved in $5 \mathrm{~mL}$ anhydrous tetrahydrofuran (THF). Oleylamine (3.5 mmol) and triethylamine $(7.1 \mathrm{mmol})$ were dissolved in anhydrous THF. They were added to the pyrenebutyryl chloride solution at 0 ${ }^{\circ} \mathrm{C}$, and this reaction mixture was stirred overnight at room temperature, dissolved in diethyl ether, and washed with copious amounts of water. The resulting organic layer was extracted and dried over sodium sulphate. Diethyl ether was evaporated, and the product was dried in vacuo at room temperature. The CNTs (300 $\mathrm{mg})$ and the oleylaminefunctionalized pyrene were dissolved in xylene, and subsequently sonication was performed for 1 hour. All of the reactions were carried out under Schlenk conditions.

To create the CNT-PDMS composite solution, $1.3 \mathrm{~mL}$ of functionalized CNT/xylene solution was stirred with $0.5 \mathrm{~mL}$ of xylene and 1g of PDMS (MED-1000, Polymer Systems Technology Ltd, UK) and subsequently sonicated for $10 \mathrm{~s}$ at 52 W. Sonication was followed by degassing for 2 minutes. MED-1000 is a one part, acetoxy curing rubber that cures on contact with atmospheric humidity. This type of PDMS was chosen due to its good adhesion to silica glass and its biocompatibility.

\subsection{Dip coating}

Dip coating was performed on silica/silica optical fibers with core/cladding diameters of $105 / 125 \mu \mathrm{m}$ and $200 / 220 \mu \mathrm{m}$ (Thorlabs FG105LCA and FG200LEA). Buffer layers of the optical fibers were mechanically stripped, and the fibers were cleaved perpendicular to their axes. Dip coating was performed with a motorized stage. Fibers were inserted into the composite solution at $2.8 \mathrm{~mm} / \mathrm{s}$, held stationary for $10 \mathrm{~s}$ and then withdrawn at the same speed. After dipping, the coatings were cured for 12 hours at $22{ }^{\circ} \mathrm{C}$, facing down. One set of optical fibers was pre-treated by immersing the tips in piranha solution ( 1 part $\mathrm{H}_{2} \mathrm{O}_{2}$ to 3 parts $\mathrm{H}_{2} \mathrm{SO}_{4}$ ) for several minutes prior to dip coating.

The coated optical fiber tips were visualized with scanning electron microscopy (SEM) and an optical stereo microscope. Their optical transmission at $1064 \mathrm{~nm}$ was measured using a photodetector and an uncoated optical fiber as a reference (5 different optical fibers of each core diameter).

A fiber coupled Nd:YAG laser was used to generate ultrasound from the dip coated optical fibers. This laser had a wavelength $1064 \mathrm{~nm}$, a pulse width of $2 \mathrm{~ns}$, and a repetition rate of $1 \mathrm{kHz}$ (SPOT-10-500-1064, Elforlight, UK). Prior to fiber coupling, laser light was transmitted through a Brewster window mounted onto a rotating stage that provided variable attenuation. The pulse energies of light delivered to the coatings were $3.6 \mu \mathrm{J}$ and $11.4 \mu \mathrm{J}$ for the $105 \mu \mathrm{m}$ core fibers and the $200 \mu \mathrm{m}$ core fibers, respectively, which corresponded to fluences of $41.6 \mathrm{~mJ} / \mathrm{cm}^{2}$ and $36.3 \mathrm{~mJ} / \mathrm{cm}^{2}$.

\subsection{Ultrasound pressure measurements}

Ultrasound pressure measurements were performed with the dip coated optical fibers positioned in water, facing a hydrophone that was positioned on the optical fiber axis. The hydrophone was a piezoelectric needle hydrophone (SN 493, Precision Acoustics, UK), with an active area diameter of $75 \mu \mathrm{m}$ and a calibration range of 1 to $30 \mathrm{MHz}$. The distance between the dip coated optical fiber end face and the active area of the needle hydrophone varied from 2 to 16 $\mathrm{mm}$.

\subsection{Tissue reflectance and imaging}

To obtain reflectance measurements, a dip coated optical fiber with a core diameter of $200 \mu \mathrm{m}$ was positioned adjacent to an optical fiber hydrophone that received ultrasound, ${ }^{13}$ with both fibers facing the sample (Figure 1). These fibers were separated by approximately $1 \mathrm{~mm}$ (center-to-center). They were mounted on a translation stage that allowed for fine 
manual positioning in three dimensions. The optical fiber hydrophone was interrogated by a console. This console produced a signal that was digitized with 14-bit resolution at $100 \mathrm{MS} / \mathrm{s}$ by a DAQ card (PCI-5122, National Instruments, Austin, USA), with acquisitions temporally synchronized to the light pulses that generated ultrasound. Optical fiber hydrophone signals were averaged over 100 consecutive acquisitions. The sample was a portion of the left ventricle of a sheep heart placed in a water bath, with the external surface of the ventricle oriented upward so that it faced the optical fibers. In the region from which reflectance measurements were obtained, there was a thin layer of adipose tissue overlying the myocardial tissue. By manually translating the optical fibers to different positions above the tissue surface and concatenating the optical hydrophone signals that were acquired from these positions, a B-mode ultrasound image was created.

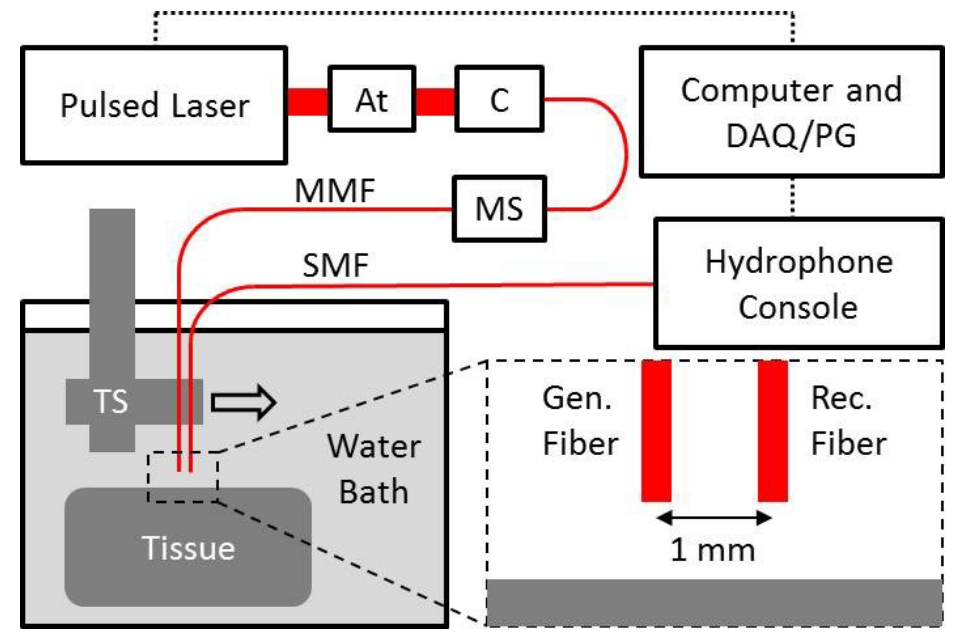

Figure 1. Experimental setup used to obtain ultrasound reflectance measurements from biological tissue. At: free-space optical beam attenuator; C: fiber coupler; MS: mating sleeve for optical fibers; MMF: multi-mode fiber; SMF: single-mode fiber; TS: translation stage; Gen. Fiber: ultrasound generation fiber; Rec. Fiber: ultrasound receiving fiber; DAQ: digital acquisition; PG: pulse generator.

\section{RESULTS}

\subsection{CNT dissolution in xylene}

The functionalized CNTs formed a stable solution in xylene, whereas the original, non-functionalized CNTs were insoluble and sedimentation was apparent within several minutes of mixing (Figure 2).

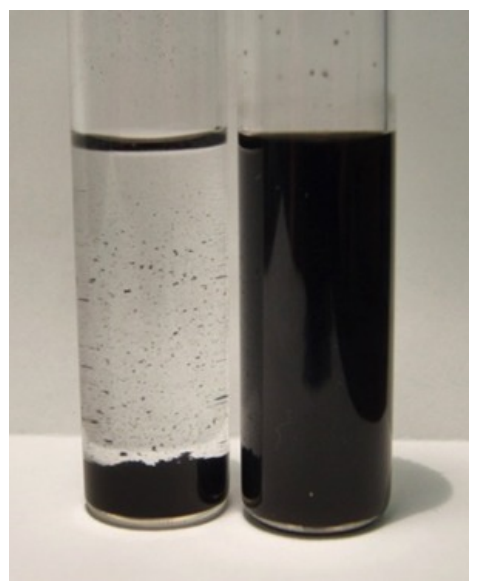

Figure 2. Non-functionalized (left) and functionalized (right) CNTs in xylene after being left to stand for one month. 


\subsection{Dipped surfaces}

As seen on the microscope and SEM images, the dip coated surfaces were largely smooth, with a thickness that appeared to decrease slightly from the center (Figures 3,4). From these images, the coating thickness appeared to be less than 20 $\mu \mathrm{m}$, but precise measurements could not be made. The optical transmission at $1064 \mathrm{~nm}$ was in the range of 13.4 to $15.5 \%$ for the $105 \mu \mathrm{m}$-core fibers; it was in the range of 1.4 to $5.4 \%$ for the $200 \mu \mathrm{m}$-core fibers. The fibers that had been pretreated with piranha solution had similar appearances with SEM to those that were not pre-treated (Figure 4). The former were much more brittle than the latter, however.
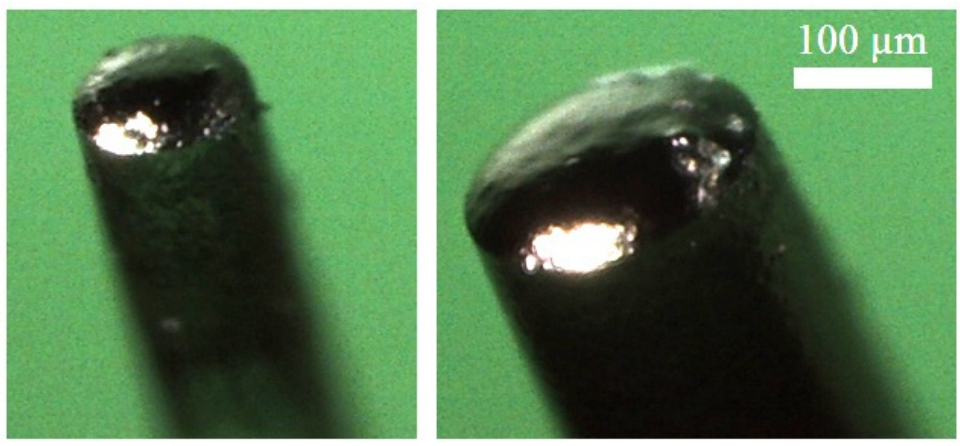

Figure 3. Dip coated optical fibers, as visualized with a stereo microscope. Left: $105-\mu \mathrm{m}$ core; right: $200-\mu \mathrm{m}$ core.
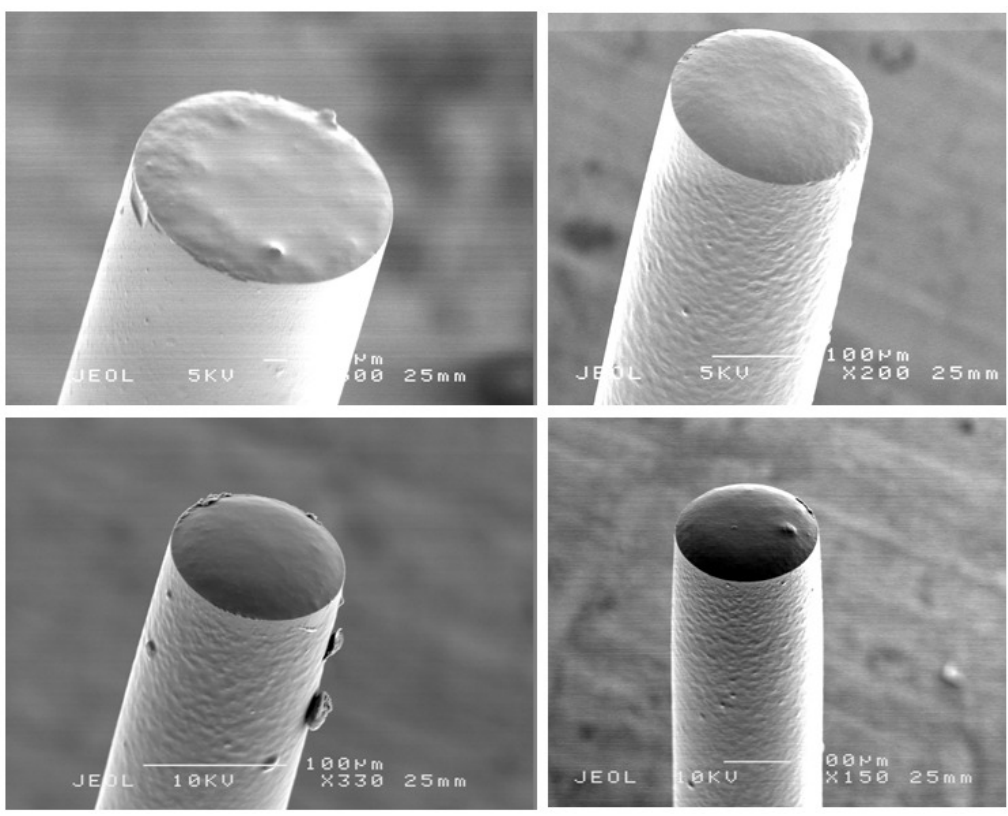

Figure 4. Dip coated optical fibers, as visualized with a scanning electron microscopy. Left: $105-\mu \mathrm{m}$ core; right: $200-\mu \mathrm{m}$ core. The tips of the top pair of optical fibers were immersed in piranha solution prior to dip coating.

\subsection{Ultrasound pressure measurements}

The ultrasound pressure decreased monotonically with the distance between the end face of the dip coated optical fiber and the needle hydrophone (Figure 5). For the $105-\mu \mathrm{m}$ core optical fiber, it decreased from 0.27 to $0.03 \mathrm{MPa}$ over distances from 2 to $16 \mathrm{~mm}$. For the $200-\mu \mathrm{m}$ core optical fiber, it decreased from 0.81 to $0.07 \mathrm{MPa}$ over the same distance range. 


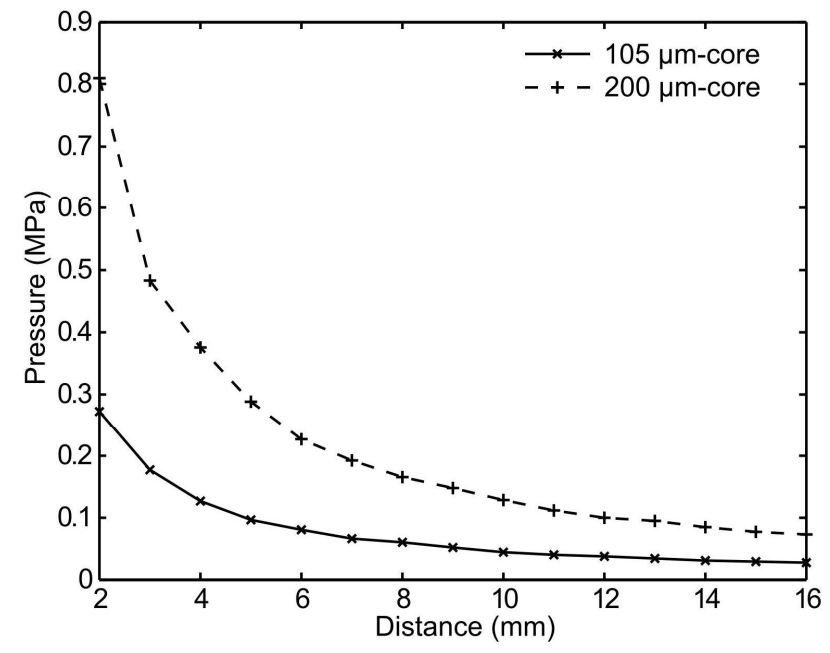

Figure 5. Ultrasound pressure generated by the dip coated optical fibers, as measured on the fiber axis with a calibrated needle hydrophone.

\subsection{Tissue sensing and imaging}

Reflections from the surface of the sheep heart tissue were apparent in the optical hydrophone signals, even when the total ultrasound propagation distance was $12 \mathrm{~mm}$ (Figure 6). The first large signal component resulted from cross-talk between the two optical fibers, in which ultrasound propagated directly from the optical transducer to the optical fiber hydrophone without reflecting from tissue. In the B-mode ultrasound image of tissue that was acquired with the optical transducer/hydrophone pair, variations in the surface height and subsurface features at depths greater than $2 \mathrm{~mm}$ were observed (Figure 7).

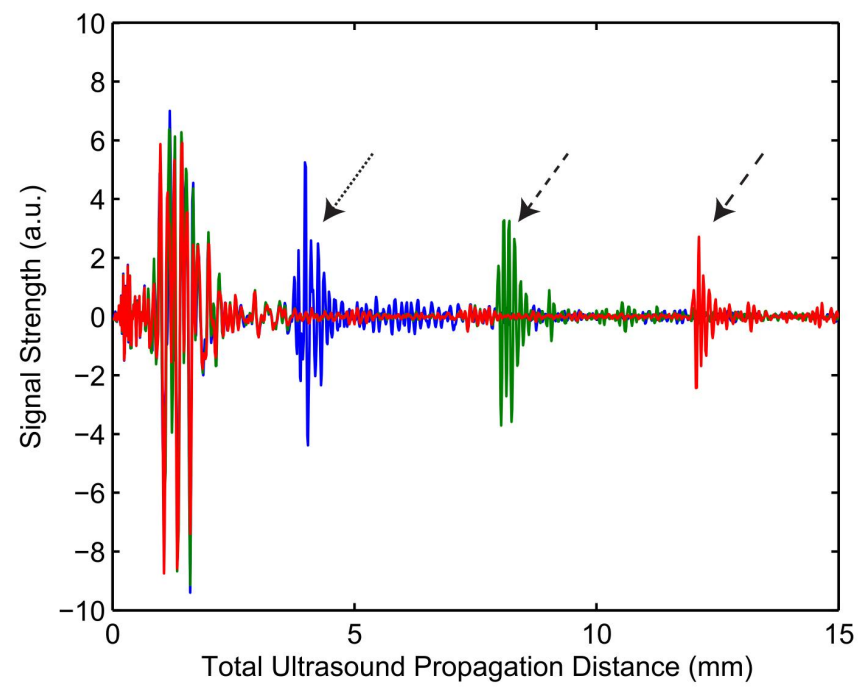

Figure 6. Ultrasound signals received from an ovine heart tissue sample, plotted against the total distance travelled by ultrasound between its generation at the dip coated optical fiber and its reception at the optical hydrophone fiber. The sample surface was positioned at distances of $2 \mathrm{~mm}$ (blue; fine-dashed arrow), $4 \mathrm{~mm}$ (green; medium-dashed arrow), and $6 \mathrm{~mm}$ (red; coarse-dashed arrow) from the end face of the optical fiber hydrophone. The signal in the range of 1 to $2.5 \mathrm{~mm}$ originated from cross-talk between the two optical fibers. 


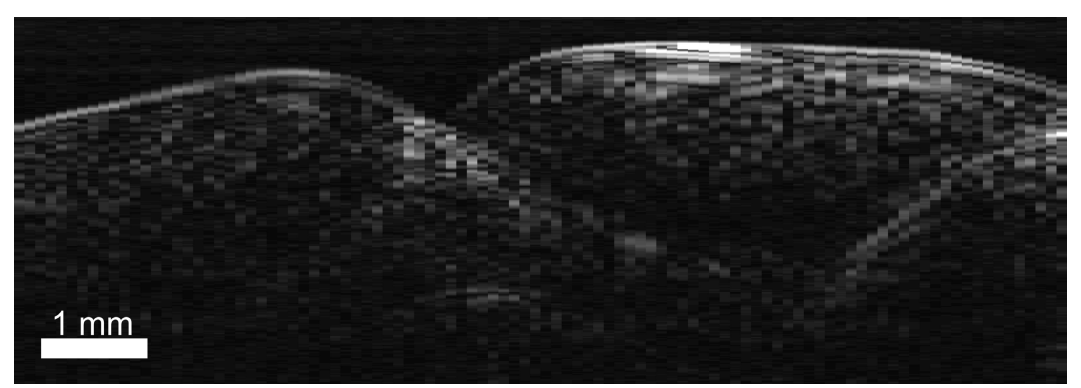

Figure 7. B-mode ultrasound image acquired from the surface of a sheep heart with a $200 \mu \mathrm{m}$ core optical fiber that was dip coated with the CNT-PDMS composite, and an optical hydrophone. This optical fiber pair was translated in one dimension relative to the tissue surface and the hydrophone signals were concatenated. The scale bar applies to both lateral and depth dimensions.

\section{DISCUSSION}

To the author's knowledge, this was the first study in which a CNT-PDMS composite coating was applied to the distal end face of an optical fiber to generate ultrasound. This type of coating provided significantly higher ultrasound amplitudes than those generated in a comparable study, in which a gold nanopore array was created on the end face of a $62.5 \mu \mathrm{m}$ core optical fiber. ${ }^{14}$ In that study, a pressure amplitude of $2.73 \mathrm{kPa}$ was measured at a distance of $1.5 \mathrm{~mm}$ from the optical fiber end face. In the study of Baac et al., ${ }^{9}$ a positive pressure of $22 \mathrm{MPa}$ was obtained with a CNT-PDMS coating, but it originated from a focused beam produced by a surface $6 \mathrm{~mm}$ in diameter.

There are several ways in which the results of this study could be extended. Optimization of ultrasound pressures with respect to the viscosity of the dip coating solution and the withdrawal speed remains to be performed. Additionally, as alternatives to CNTs, carbon black or graphene could be functionalized to enhance their solubility in organic solvents of PDMS.

The ultrasound reflectance measurements and the B-mode ultrasound image that were acquired optically are promising from a medical imaging standpoint. This study provided only a preliminary indication of the potential of all-optical Bmode ultrasound imaging using optical fibers. Performance metrics such as the depth-dependence of the spatial resolution remain to be measured. It is likely that significant improvements in performance could be achieved by optimizing the energies and repetition rates of the light pulses that generated ultrasound.

In this study, a composite of CNTs and PDMS was created by functionalizing CNTs and dissolving them into xylene, and subsequently dissolving the CNT-xylene solution into PDMS. Using dip coating, the CNT-PDMS composite was applied to the end faces of optical fibers for optical ultrasound generation. The optical transducers that were created may offer significant advantages over electrical transducers in terms of simplicity of fabrication and ease of integration into miniature medical imaging probes.

\section{REFERENCES}

[1] Li, X., Wu, W., Chung, Y., Shih, W.Y., Shih, W.-H., Zhou, Q., and Shung, K.K., "80-MHz intravascular ultrasound transducer using PMN-PT free-standing film," IEEE Transactions on Ultrasonics, Ferroelectrics, and Frequency Control 58(11), 2281-2288 (2011).

[2] Degertekin, F.L., Guldiken, R.O., and Karaman, M., “Annular-ring CMUT arrays for forward-looking IVUS: transducer characterization and imaging," IEEE Transactions on Ultrasonics, Ferroelectrics, and Frequency Control 53(2), 474-482 (2006).

[3] Hou, Y., Kim, J.-S., Ashkenazi, S., Huang, S.-W., Guo, L.J., and O’Donnell, M., "Broadband all-optical ultrasound transducers," Applied Physics Letters 91(7), 073507 (2007).

[4] Buma, T., Spisar, M., and O’Donnell, M., "High-frequency ultrasound array element using thermoelastic expansion in an elastomeric film," Applied Physics Letters 79(4), 548-550 (2001). 
[5] Acquafresca, A., Biagi, E., Masotti, L., and Menichelli, D., "Toward virtual biopsy through an all fiber optic ultrasonic miniaturized transducer: a proposal," IEEE Transactions on Ultrasonics, Ferroelectrics, and Frequency Control 50(10), 1325-1335 (2003).

[6] Swift, C.I., Pierce, S.G., and Culshaw, B., "Laser generated ultrasound using directly coated fibre optic patchcords," Electronics Letters 36(25), 2113-2114 (2000).

[7] Lee, S.H., Park, M., Yoh, J.J., Song, H., Jang, E.Y., Kim, Y.H., Kang, S., and Yoon, Y.S., "Reduced graphene oxide coated thin aluminum film as an optoacoustic transmitter for high pressure and high frequency ultrasound generation," Applied Physics Letters 101(24), 241909 (2012).

[8] Baac, H.W., Ok, J.G., Park, H.J., Ling, T., Chen, S.-L., Hart, A.J., and Guo, L.J., "Carbon nanotube composite optoacoustic transmitters for strong and high frequency ultrasound generation," Applied Physics Letters 97(23), 234104 (2010).

[9] Baac, H.W., Ok, J.G., Maxwell, A., Lee, K.-T., Chen, Y.-C., Hart, A.J., Xu, Z., Yoon, E., and Guo, L.J., "Carbon-nanotube optoacoustic lens for focused ultrasound generation and high-precision targeted therapy," Scientific Reports 2, 989 (2012).

[10] Lipomi, D.J., Martinez, R. V., Kats, M.A., Kang, S.H., Kim, P., Aizenberg, J., Capasso, F., and Whitesides, G.M., "Patterning the tips of optical fibers with metallic nanostructures using nanoskiving," Nano Letters 11, 632-636 (2011).

[11] Kuroda, M., and Nishino, T., "Preparation of tips coated with poly(dimethylsiloxane) for scanning tunneling microscopy in aqueous solutions," The Review of Scientific Instruments 82(6), 063707 (2011).

[12] Majeed, S., Filiz, V., Shishatskiy, S., Wind, J., Abetz, C., and Abetz, V., "Pyrene-POSS nanohybrid as a dispersant for carbon nanotubes in solvents of various polarities: its synthesis and application in the preparation of a composite membrane," Nanoscale Research Letters 7(1), 296 (2012).

[13] Zhang, E.Z., and Beard, P.C., "A miniature all-optical photoacoustic imaging probe," in Proc. SPIE Vol. 7899 7899, A. A. Oraevsky and L. V. Wang, Eds., 78991F (2011).

[14] Tian, Y., Wu, N., Zou, X., Felemban, H., Cao, C., and Wang, X., "Fiber-optic ultrasound generator using periodic gold nanopores fabricated by a focused ion beam," Optical Engineering 52(6), 065005 (2013). 